\title{
ALPHA-PARTICLE LOSSES IN COMPACT TORSATRON REACTORS *
}

\author{
S. L. Painter**, J. F. Lyon \\ Fusion Energy Division, Oak Ridge National Laboratory \\ Oak Ridge, Tennessee, USA
}

ABSTRACT Loss of alpha particles in compact torsatron rcactors is studicd. For 6 , 9. and 12 field period reactors, the direct loss is a relatively weak function of radius and energy and varies from $\simeq 33 \%$ for $M=6$ to $=18 \%$ for $M=12$. Loss of alpha particles through scattering into the loss region is calculated using the Fokker-Plank equation for fast ions and found to contribute an additional alphaparticle energy loss of $\simeq 15 \%$. The consequences of these relatively large losses for torsatron reactor design are discussed.

The relationship between the direct particle losses and the magnetic field structure is also studied. Orbit losses from a variety of stellarator configurations are calculatcd and a figure-of-merit that characterizes the orbit confinement of a magnetic configuration is deduced from these calculations. This figure-of-merit is used to show how the direct losses might be reduced at low aspect-ratio. Effects of finite beta on the direct particle losses are also addrcsscd, and are shown to significantly incrcase the direct losses in some configurations.

The compact torsatron sequence ${ }^{1}$ is a family of low aspect-ratio $l=2$ torsatron configurations optimized for high-beta operation. These configurations make interesting reactor candidates because they have the capablity for high-beta operation in the second stability regime, possess natural divertors, and have relatively open coil geometry for access to the plasma. Compaci torsatrons are, however, optimized for MHD properties, not orbit confinement. A large fraction $\left(\approx \frac{1}{3}\right)$ of particles in these devices are trapped in the relatively large helical ripple in the magnetic field strength $B$. Toroidal effects at low aspect ratio cause orbits of these trapped particles to deviate significantly from flux surfaces leading to increased transport and in some cases to loss of the particles from the confinement region. Radial electric fields which develop to maintain quasineutrality of the plasma can reduce the deviation of the trapped particles from the flux surface through $E \times B$ orbit rotation, but only for those particles which have kinetic energies less than their potential energies

- Research sponsored by the Office of Fusion Energy, L.S. Department of Energy, under contract DE-AC05-840R21400 with Martin Marietta Energy Systems, Inc.

* Research performed to meet requirements for the degree of PhD in Nuclear Engineering, Lniversity of Tennessee. 
in the electric field. Energetic particles created by fusion reactions or through external plasma heating will not be affected by radial electric fields and many of these energetic particles may be lost from the plasma region. The confinement of energetic alpha particles in compact torsatron reactor configurations is addressed in this paper.

\section{CALCULATION OF ALPHA-PARTICLE LOSSES}

The complicated magnetic field structure at low aspect ratio and the large particle energies of interest here make a numerical treatment of the particle loss fraction necessary. Direct pärticle losses were calculated by computing collisionless guiding center orbits ${ }^{2-5}$ for a distribution of alpha particles launched randomly from a given flux surface. Details of the calculation can be found in reference 6. Each particle was followed until it left the plasma volume, made two poloidal revolutions, or exceeded a preset time cutoff (several hundred helical bounce periods). Magnetic coordinates $^{2}$ were used so that only the magnitude of the nagnetic field and not its vector components was needed in the calculation. The magnetic field strength was represented by a double Fourier expansion in the poloidal $(\theta)$ and toroidal $(\phi)$ angles

$$
B(\rho, \theta, \phi)=\sum B_{n, m}(\rho) \cos (n \phi-m \theta)
$$

using 12 to 25 terms. Here the normalized radial coordinate $(\rho)$ is proportional to the square root of the toroidal flux.

\section{ALPHA-PARTICLE LOSSES FOR REFERENCE REACTORS}

The fraction of alpha particles confined is shown versus the radial coordinate $\rho$ in Fig. 1 for three minimum radius reference reactors ${ }^{\top}$, ATR-1, ATR-2 and ATR-3. ATR-3 is the ATF configuration scaled up to reactor size (aspect ratio $7.78,11.1$ meters major radius) while ATR-1 and ATR-2 are lower-aspect-ratio configurations (aspect ratio 3.87, 8.4 meters major radius for ATR-1; aspect ratio 4.66, major radius 10.5 meters for $A T R-2)$. The magnetic field strength at the magnetic axis is $5 \mathrm{~T}$ in all cases and the particle energy is $3.5 \mathrm{MeV}$. The alpha-particle gyroradius 
is $2-3 \%$ of the plasma radius. The direct particle loss fraction is a relatively weak function of radius and is sn:aller for the higher aspect ratio ATR-3. The difference in the alpha-particle loss fraction is due to a smaller trapped particle fraction for ATR3 and to better confinement of the trapped particles. ATR-3 confines $60 \%$ of the trapped particles that start at $\rho=0.25$ while ATR-1 and ATR-2 lose nearly 100\% of trapped particles starting from the same radius. This can be seen in Fig. 2 where the fraction of trapped particles lost is shown versus the pitch-angle parameter for ATR-3 and ATR-1. Deeply-trapped particles (those having small pitch angles) are confined in ATR-3 but not in ATR-1.

Alpha particles and energy are also lost when particles scatter into the direct loss region. Energetic alpha particles slow down and give up their energy to the background electrons without undergoing significant pitch-angle scattering until they reach energy $E_{c r i t} \simeq 32 T_{e}$. Below $E_{c r i t}$ pitch-angle scattering on the background ions becomes important and most alpha particles find their way into the loss region before they can slow down enough to become confined by the radial electric field, thus preventing the accumulation of helium ash. The additional energy and particle losses due to alpha particles scattering into the loss region can be estimated from known solutions to the Fokker-Plank equation for fast ions ${ }^{9-11}$. The additional energy losses estimated this way are $\approx 15 \%$, but $\approx 80 \%$ of the alpha particles are lost before thermalizing.

The total (direct plus indirect) loss of alpha heating power is $32 \%$ for ATR-3 and $46 \%$ for ATR-1 for alpha source profiles of the form $\left(1-\rho^{2}\right)^{4}$ which corresponds to parabolic density and temperature profiles. The main implications of this loss are to increase the plasma temperature at ignition and the external heating power required to reach ignition. Once ignition is reached, the alpha-particle loss region is beneficial; it aids in burn control by reducing the amount of heat available to the thermal excursion, prevents thermalized alpha particles from accumulating in the plasma, and channels the particle energy into narrow helical stripes on the outside of the torus where it can be recovered efficiently.

\section{DEPENDENCE ON THE MAGNETIC FIELD STRUCTURE}

In order to understand how the direct particle losses depend on the structure of the magnetic field and possibly how they might be reduced, the orbit confinement 
characteristics of a number of idealized stellarator configurations and a number of variant compact torsatron configurations were also investigated. In this section, the plasma radius is $2 \mathrm{~m}$, the magnetic field strength on axis is $5 \mathrm{~T}$, and the alphaparticle energy is $3.5 \mathrm{MeV}$. The idealized stellarator configurations have $B$ of the form

$$
B(\rho, \theta, \phi)=B_{0}{ }^{1} 1-\epsilon_{1 a} \rho \operatorname{ros}(\theta)+\epsilon_{r a} \rho^{2} \cos (6 \phi-2 \theta)
$$

were $\epsilon_{h a}$ is the helical ripple at the plasma edge and $\epsilon_{1 a}$ is the inverse plasma aspect ratio. The fraction of trapped alpha particles lost versus the ratio $p_{2}=\epsilon_{h a} c_{1 a}$ is shown in Fig. 3 for aspect ratios 5 and 10. The fraction of trapped particles Inst is approximately independent of aspect ratio for a given value of $p_{2}$, is nearly unity when $p_{2}=1$, and decreases on either side of $p_{2}=1$.

The results shown in Fig. 3 can be understood on the basis of the $B_{m+n}$ model. $B_{\min }$ surfaces are surfaces of constant

$$
B_{\min }(\rho, \theta)=\min _{\phi}[B(\rho, \theta, \phi)]
$$

and in the absence of radial electric fields are good approximations to deeply-trapped particle orbits. Particles that are not deeply trapped also tend to drift along B-min contours so the confinement of a distribution of alpha particles (not all deeplytrapped) is correlated with the closing of these contours. When $p_{2}=1, B_{\min }$ contours are not closed in the plasma region and helically-trapped particles drift out of the confinement region. When $p_{2}>1$, the configuration appears nearly helically symmetric, $B_{\min }$ contours coincide approximately with flux surfaces, and helically-trapped particles are confined. In the case $p_{2}<<1$ the alplia-particle confinement is not correlated with closing of the $B_{\min }$ contours because the majority of trapped particles are trapped by the toroidal $\frac{1}{R}$ variation in $B$ and not by the helical variation. In this case the dominant loss mechanism is stochastic drift ${ }^{12}$ of the banana orbits.

The confinement of trapped particles in more complicated magnetic fields can also be explained with the $B_{\min }$ model. To illustrate this, trapped particle loss fractions were computed for a variety of torsatron configurations. These configurations differ from the reference reactors in that they have different vertical fields, different helical coil winding trajectories, or $l=1$ coils in addition to the primary $l=2$ coil set. Aspect ratios ranging from 3 to 8 and helical ripples ranging from 0.1 to 0.4 were 
considered. The results of these calculations are summarized in Fig. 4. The vertical axis is the fraction of trapped particles lost; the horizontal axis is the fractional area $f$ of closed $B_{m a n}$ contours. The fraction of trapped particles confined can be fitted by

$$
\begin{array}{rlrl}
f_{c}=1.61 f^{2} & f<0.79 \\
f_{c}=1.0 & f>0.79
\end{array}
$$

with an rms error of only $8^{\infty}$ for the wide variety of vacuum configurations consid. ered.

\section{REDLCTION GF DIRECT LOSS FRACTION}

The correlation bet ween the area of closed $B_{\min }$ contours and the direct loss fraction can be used to study how additional helical harmonics might be added to the single helicity idealized configuration of (Eq. 2) in order to reduce the loss fractions. Flux surfaces are not guaranteed to exist for these model configurations; the results presented here are, however, uselul for understanding the tradeoffs available to the stellarator reactor designer.

Mynick'14 proposed poloidal modulation of the helical ripple

$B(\rho, \theta, \phi)=B_{0}\left\{1-\epsilon_{\mathrm{ta}} \rho \cos (\theta)+[1-\sigma \cos (\theta)] \epsilon_{h a} \rho^{2} \cos (6 \phi-2 \theta)\right\}$

which is the same as (Eq. 2) with $\epsilon_{h a}$ replaced by $[1-\sigma \cos (\theta)] \epsilon_{h a}$. Here $\sigma$ is an optimization parameter which is chosen to make trapped particle orbits coinicide approximately with flux surfaces. Mynick took $p_{2}=1$ and found optimized configurations with $\sigma=1$. Using the correlation between the $B_{\min }$ area and losses, it can be shown that this configuration is a member of a family of zero loss configurations having $\sigma \approx 1 / p_{2}$.

If (Eq. 4) is rewritten in terms of the Fourier expansion (Eq. 1), a spectrum of helical harmonics results. Perhaps a more straightforward way of improving orbit confinement is to add only $l=1$ and $l=3$ satelite harmonics to the single helicity configuration. Contours of constant particle loss fraction found with the correlation (Eq. 3) for a configuration having $p_{2}=1$ are shown in Fig. 5. Here $p_{1}$ and $p_{3}$ are $l=1$ and $l=3$ ripple amplitudes normalized to the inverse plasma aspect ratio similiar to the definition of $p_{2}$. For single helicity configurations having $p_{2}=1$, the 
trapped particle loss fraction is unity. Additional $l=1$ and $l=3$ terms reduce the loss fraction to zero in a manner similiar to Mynick's $\sigma$-optimized configuration. In these configurations, the $B_{m \text { m }}$ contours coincide approximately with Hux surfaces and the configuration appears helically symmetric to helically-trapped particles.

\section{EFFECTS OF FINITE BETA}

Vacuum field configurations which approximate the zero loss configurations discussed above can be produced by the ATR-3 coil set by including additional vertical fields to change the shape of the vacuum flux surfaces or shift the magnetic axis inward in major radius. Trapped particle loss fractions can then be reduced to zero. However, Pfirsch-Schluter currents which flow in nonzero-beta plasmas can alter the $B$ spectrum and change particle orbits. To study this eifect. three sequences of fixed-boundary zero net current equilibria were obtained with the 3 $D$ code MMEC $^{14}$. The initial racuum field configurations for the three sequences are the standard ATR-3, ATR-3 shifted inward, and ATR-3 with increased central transform. The latter two vacuum configurations have zero losses. The fraction of trapped particles lost versus volume averaged beta is shown in Fig. 6 for the three sequences. The loss fraction increases toward unity with increasing plasma beta. At volume-averageri beta of a few percent, the reduction in losses gained by shifting or shaping the vacuum field flux surfaces has disappeared. This behaviour can also be understood within the context of the $B_{\min }$ model. As beta increases and the magnetic axis shifts outward relative to the fixed helical coil set, the $B_{\min }$ contours become distorted and noncircular. Even though the area of closed $B_{\min }$ contours does not decrease substantially, the center of the $B_{\min }$ contours shifts relative to the magnetic axis (Fig. 7). This causes deeply-trapped particle orbits to pass close to the plasma boundary and particles which are not deeply-trapped leave the confinement region. $B_{\min }$ contours for two values of plasma beta are shown in Fig. 8 for the inward shifted ATR-3.

The sequence of finite beta equilibria used here are intended to illustrate how finite beta can undo orbit optimization; they are not intended to represent a reactor startup scenario. On-going studies seek to identify startup sequences for ATR-3 which have improved orbit confinement. This ancilliary optimization with vertical fields is not effective at improving orbit confinement in the lower aspect ratio ATR- 
1 and ATR-2 however. In order to find low aspect-ratio configurations with good orbit confinement, it will be necessary to int roduce transport considerations directly into the primary configuration optimization procedure ${ }^{15}$.

\section{ACKNOMLEDGMENTS}

The authors acknowledge $C$. L. Hedrick and B. A. Carreras for suggesting the correlation between alpha-particle losses and the area of closed $B_{\operatorname{man}}$ contours and ihe addition of $l=1$ and $l=3$ components. They also thank $l$. E. Lyuch for providing Fourier components of $B$ for the configurations studied, R. H. Fowler for providing the orbi-following code, and $N$. Dominguez for providing the finite-beta equilibria. One au hor (SLP) also thanks P. N. Stevens for his encouragement.

\section{REFERENCES}

1. B. A. CARRERAS, N. DOMINGUEZ, L. GARCIA, V. E. LYNCH, J. F. LYON. J. R. CARY, J. D. HANSON, and A. P. NAWARRO, "Low-Aspect-Ratio Torsation Configurations," Nucl. Fusion, 28, 1195 (1988); see also ORNL/TM-10030, Oak Ridge National Laboratory (1987).

2. A. H. BOOZER, "Guiding Center Drift Equations," Phys. Fluids, 23, 904 (1980).

3. G. KLO-PETRAIIC, A. H. BOOZER, J. A. ROME, and R. H. FOWLER, "Numerical Evaluation of Magnetic Coordinates for Particle Transport Studies in Asymmetric Plasmas," J. Comput. Phys., 51, 261 (1983).

4. A. H, BOOZER and G. KLO-PETRAVIC, "Monte Carlo Evaluation of Transport Coefficients," Phys. Fluids, 24, 851 (1981).

5. R. H. FOWLER, J. A. ROME, and J. F. LYON, "Monte-Carlo Studies of Transport in Stellarators," Phys. Fluids, 28, 338 (1985).

6. S. L. PAINTER, J. F. LYON, "Alpha Particle Losses in Compact Torsation Reactors," Fusion Technol, accepted for publication; see also ORNL/TM1-10792, Oak Ridge National Laboratory (Oct. 1988). 
7. J. F. LYON, B. A. CARRERAS, V. E. LYNCH, J. S. TOLLIVER, and I. N. SIIATOSLAVSKY, "Compact Torsatron Reactors," Fusion Technol, 15, 1401 (1989).

8. J. F. LION, B. A. CARRERAS, K. K. CHIPLEY, M. J. COLE, J. H. HARRIS, T. C. JERNIGAN, R. L. JOHNSON, V. E. LYNCH, B. E. NELSON, J. A. ROME, J. SHEFFIELD, and P. B. THOMPSON, "The Advanced Toroidal Facility," Fusion Technol, 10, 179 (1986).

9. J. G. CORDEY and W. G. F. CORE, "Energetic Particle Distribution in a Toroidal Plasma with Neutral Injection Heating," Phys. Fluids, 17, 1626 (197f).

10. J. A. ROME, D. G. McALEES, J. D. CALLEN, and R. H. FOWLER, "ParticleOrbit Loss Regions and Their Effects on Neutral-Injection Heating in Tokamaks," Nucl. Fusion, 16, 55 (1976).

11. D. ANDERSON, H. HAMNÉN, and M. LISAK, " $a$-Particle Ripple Losses During Slow Down in a Tokamak Reactor," Phys. Fluids, 25, 353 (1982).

12. R. J. GOLDSTON, R. B. WhITE, and A. H. BOOZER, "Confinement of High-Energy Trapped Particles in Tokamaks," Phys. Rev. Lett., 47, 647 (1981).

13. H. E. MYNICK, T. K. CHU, and A. H. BOOZER, "Class of Model Stellarator Fields with Enhanced Confinement," Phys. Rev. Lett., 48, 322 (1982).

14. S. P. HIRSHMAN and J. C. WHITSON, "A Steepest-Descent Moment Method for Three-Dimensional Magnetohydrodynamic Equilibria," Phys. Fluids, 26, 3553 (1983).

15. S. P. HIRSHMAN, R. N. MORRIS, C. L. HEDRICK, J. F. LYON, R. H. FOWLER, J. A. ROME, S. L. PAINTER, W. L. VAN RIJ, "Low-AspectRatio Optimization Studies for ATF-II, " these procedings.

\section{DISCLAIMER}

This report was prepared as an account of work sponsored by an agency of the linited States Government Neither the United States Government nor any agency theresf, nor any of their emplovees, makes any warranty express or implied, or assumes any legal lıabilıty or responsıbility for the accuracy. completencss, or usefulness of any information, apparatus, product, or process disclosed, or represents that $1 t 5$ use would not infringe privately owned rights. Reference herein to any specific commercial prinduct, process, or servise by trade name. trademark. manufacturer, or otherwise does not necessarily constitute or unply its endorsement, recommendation, or favoring by the United saies Government or any agency thereof The vews and opinions of authors expressed herein do not necessarily state or reflect those of the Uniled States Government or any agency thereof. 

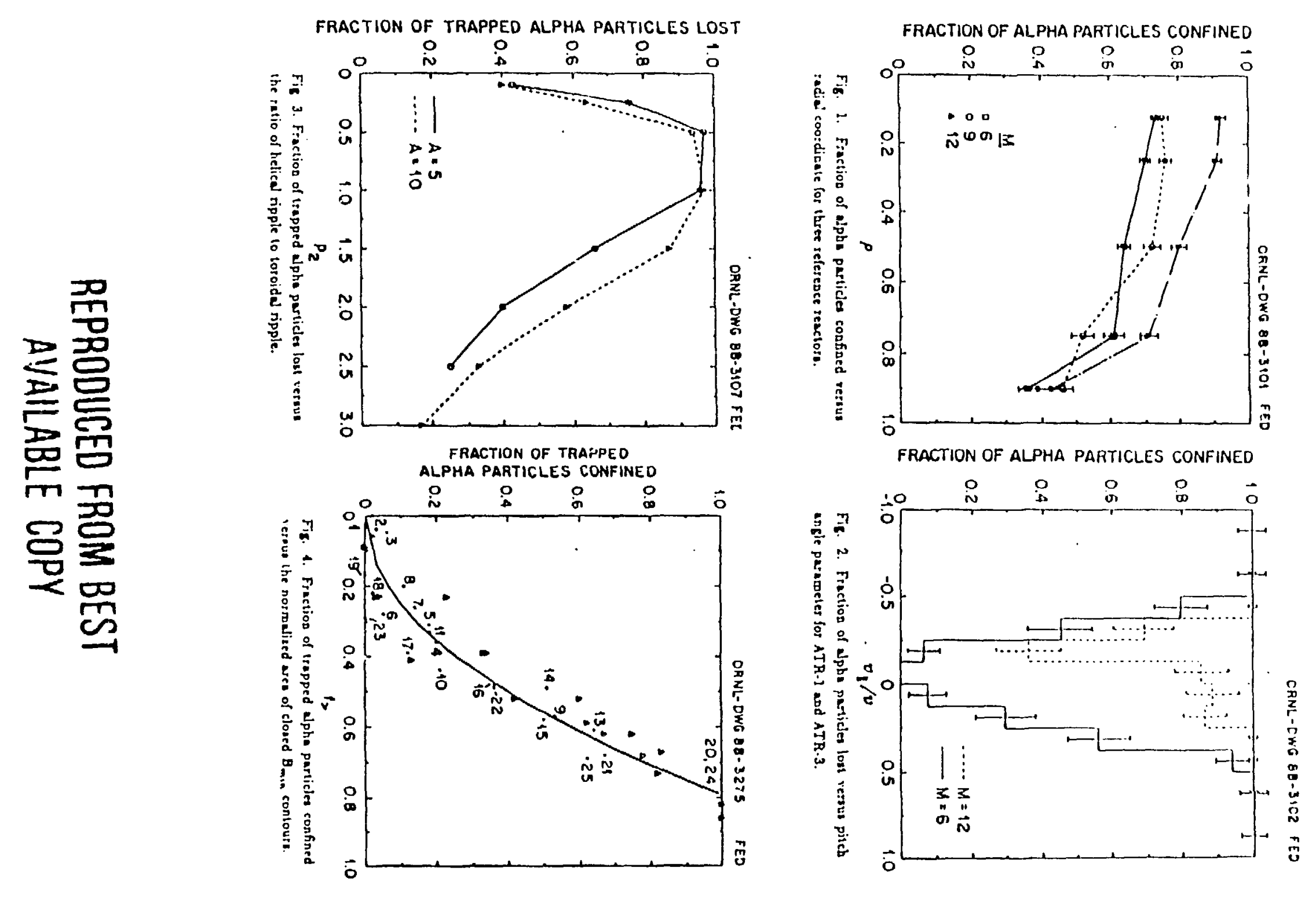


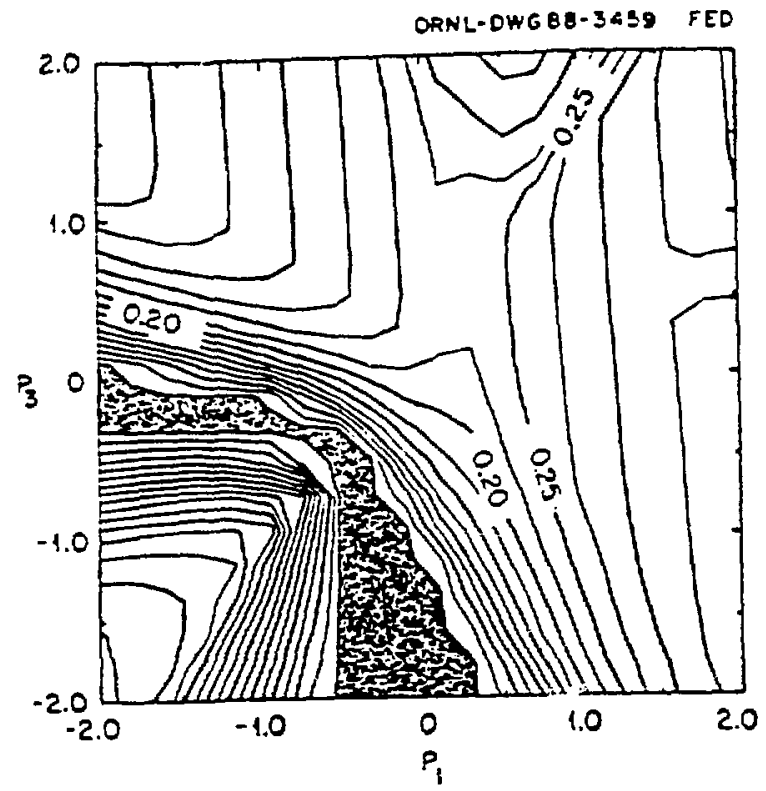

Fig. 5. Conlouss of consiad dpha particle losses for recuum field atelleretor haring apect ratio 5 and helical ripple of 0.2 . $p_{1}$ and $p_{2}$ are $l=1$ and $l=3$ ripple amplitudes no:melize to the inve:sr aspeet satio.

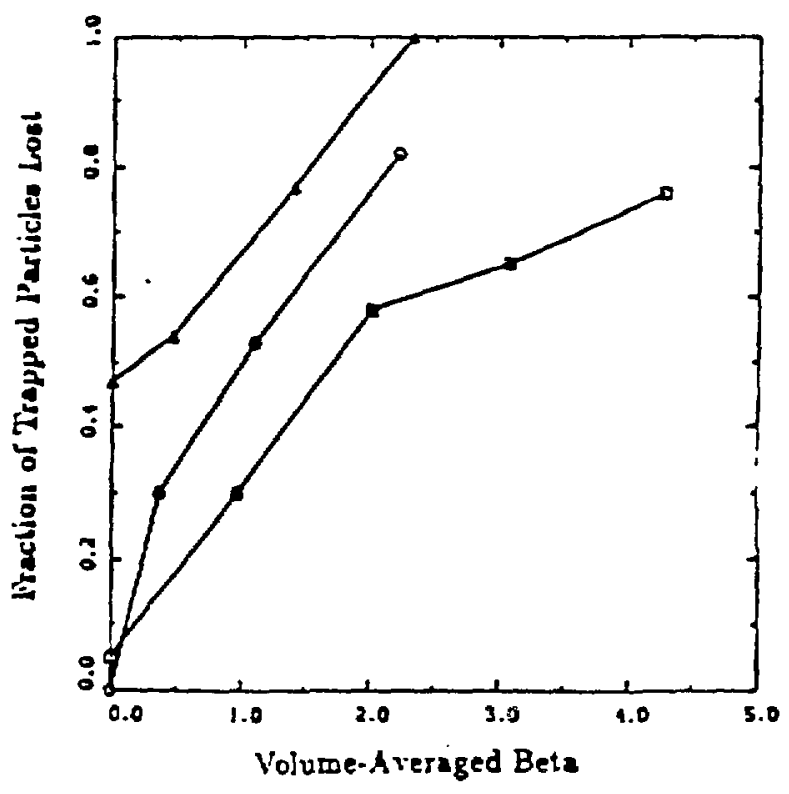

Fig. 6. Frartion of irapped ajpla particle lost vesuve roluree-eresaged bets for three rarients of ATR-3. Trianglea represeat tbe uleodad configuration, iquarea we for a conforsation ahifled inrard in major radiua, and circla are for a conffuration witb increased cen. tral irnoifores.

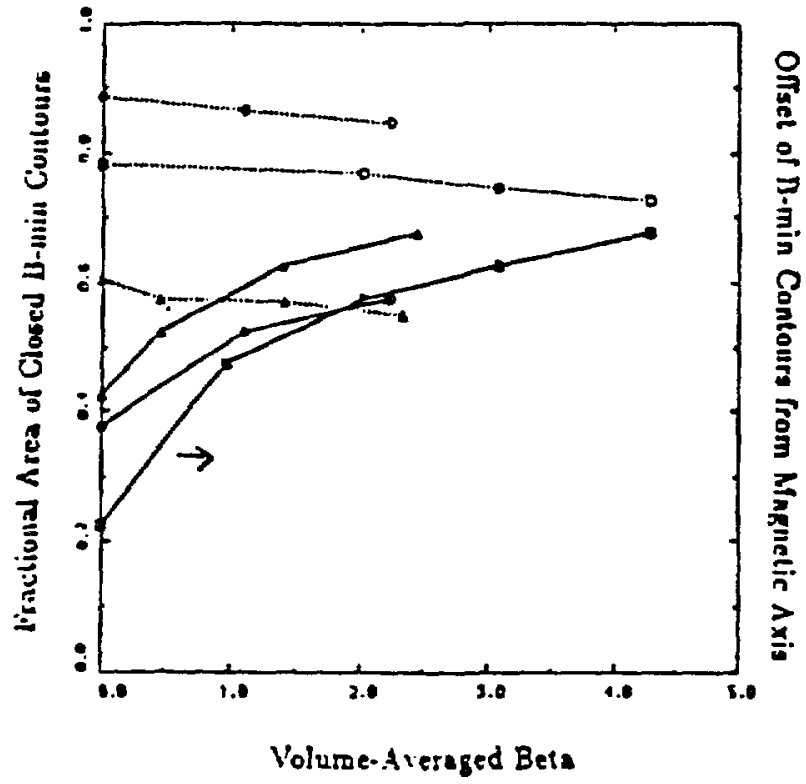

Fis. 7. Variation of the fractiod ares of closed $B_{\text {min }}$ contours and the offel of the $B_{\min }$ contous from the magnetic axis witb volume-veraged be!s for thre rarisnis of ATR.3. The symbols denole the une configutions whese in Fis. 6.

\section{REPRODUCED FROM BEST AVAILABIEE COPY}



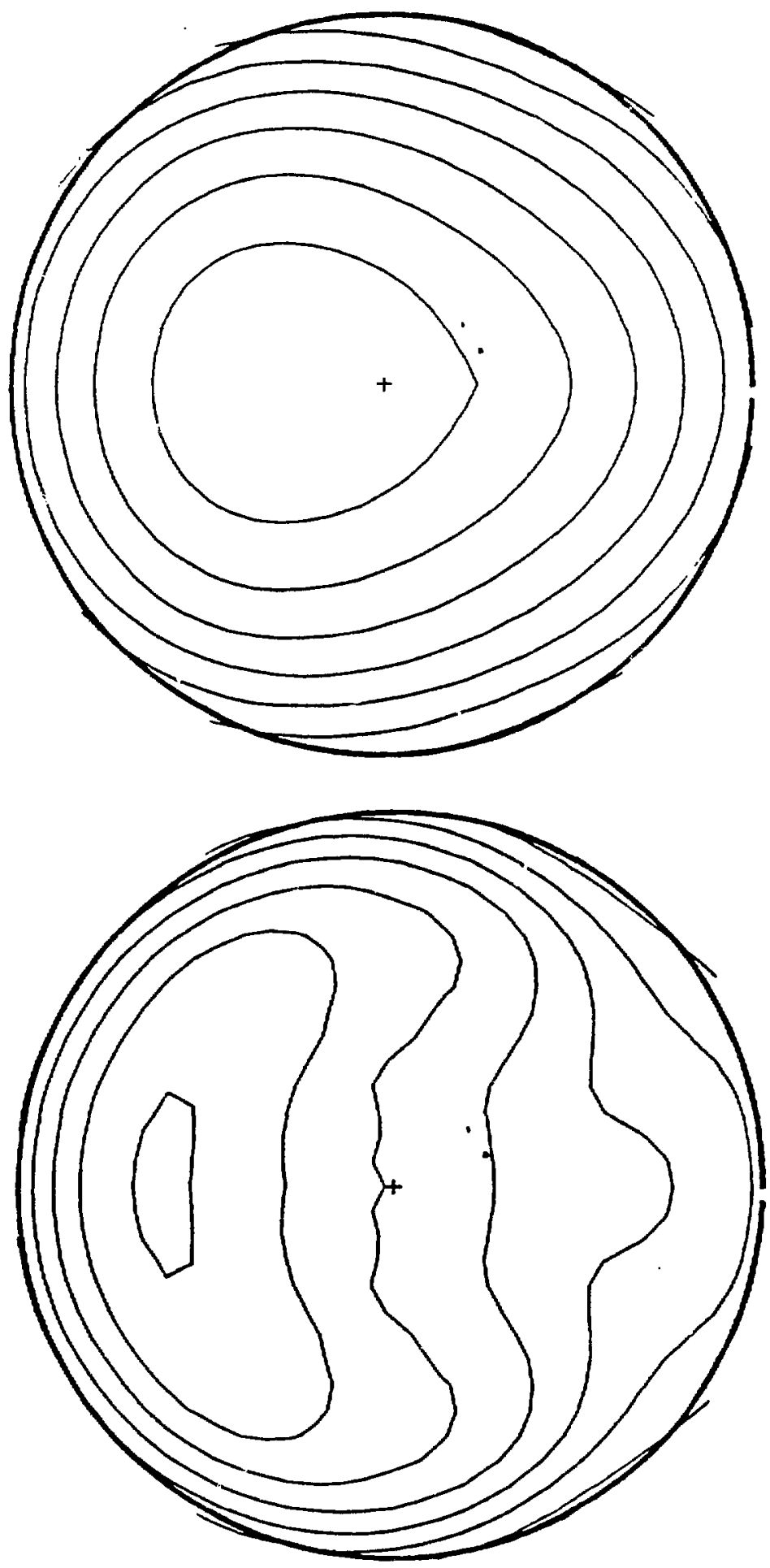

Fig. 8. $B_{\min }$ contours at $\beta=0$ and $\beta=3 \%$ for the shifted-in ATR-3 configuration. The radial coordinate is proportional to the square root of toroidal flux and the angle variable is Boozer's poloidal angle. 